\title{
HUMANIZACIÓN DEL CUIDADO DE ENFERMERÍA DURANTE EL PARTO
}

\section{HUMANIZATION IN THE INFIRMARY'S CARE TAKE DURING CHILDBIRTH}

Rosa Coral Ibarra ${ }^{1}$

\section{RESUMEN}

A lo largo de la trayectoria profesional en diferentes instituciones de salud y durante la experiencia de estar en contacto con las mujeres en trabajo de parto, se han reportado quejas de maltrato por parte del personal de salud. Esto ha sido corroborado por profesionales y estudiantes de enfermería en contacto con las gestantes y quienes refirieron haber observado actitudes de intolerancia, mal genio, incomprensión y expresiones inadecuadas. De acuerdo a lo anterior surgió la necesidad de desarrollar un trabajo de investigación, cuyo objetivo general fue el de elaborar un modelo de atención de enfermería, con énfasis en el aspecto humanístico del cuidado a la mujer durante el trabajo de parto y parto, basado en fundamentos teóricos, resultados de un proceso de investigación y experiencias del investigador en esta área. La investigación, se llevó a cabo en el Departamento de Cundinamarca (Colombia) en una empresa social del estado de II nivel El estudio fue de tipo descriptivo, se tomó una muestra de 80 mujeres a quienes se les aplico una encuesta en la etapa de posparto. Los resultados indicaron que del total de la muestra analizada, el $41 \%$ reconocieron a la enfermera, el $38 \%$ no la reconocieron y el $11 \%$, a pesar de haberla identificado no tuvieron ningún contacto con ella. De las 33 mujeres que identificaron a la enfermera, solo 24 recibieron algún tipo de cuidado, hecho que justificó la elaboración del modelo de humanización, que aplica mas específicamente a la institución donde se realizó el estudio.

Palabras clave: Modelo, humanización, cuidado, enfermería, parto.

${ }^{1}$ Enfermera, M.Sc. Docente UDCA. rocoral1@hotmail.com. Dirección para correspondencia: Calle 222 No 54-37 Bogota, D.C.
SUMMARY

Permanent complaints have been received at different health institutions from women at childbirth regarding attention by the health-care personnel. Such information concerning mistreatment or neglect, intolerant or inconsiderate attitudes, bad temper and impoliteness has been corroborated by infirmary students and professionals in contact with pregnant women. In order to asses this aspects, it was determined to carry out a study in a level II health-care institution, located in the Department of Cundinamarca (Colombia), with the prime objective of creating a health model with particular emphasis in the care of the mother during work of childbirth and childbirth, based on theoretic fundaments, result of a research process and experiences of the investigator in this area. The information for the study was gathered among a group of 80 women in puerperium stage, entering the hospital pursuing medical attention. From the surveyed females, $41 \%$ identified the nurse, $38 \%$ did not identify him/her and $11 \%$ identified the nurse, but did not have any contact with him/her. Only 24 women out of the 33 who identified the nurse, received some type of care. These results justified the elaboration of a humanization model of infirmary care for pregnant women, previous to, during, and after childbirth, that applies more specifically to the institution where the studio was carried out.

Key words: Model, humanization, care, infirmary, childbirth.

\section{INTRODUCCIÓN}

El cuidado de enfermería durante el parto requiere de consideraciones especiales hacia la mujer, ya que en esta etapa se atraviesa por una serie de cambios, 
tanto biológicos como de tipo emocional o social. La madre entra en un proceso de adaptación en donde las influencias culturales, el apoyo familiar, la intervención del equipo de salud y, específicamente, el de enfermería, juegan un papel importante en la evolución satisfactoria de este proceso.

El hablar de humanización lleva consigo la particularidad de que los seres humanos son únicos e irrepetibles y, por lo tanto, se deben tener en cuenta las individualidades para la atención; de acuerdo con Trevizan et al. (2003), el profesional de enfermería es el responsable de gerenciar la atención a las personas con apoyo sustancial en la propuesta con visión humanista. Al respecto, en una investigación realizada por Da Silva et al. (2002), titulada "Cuidados de enfermería un sentido para enfermeras y pacientes", realizado en la ciudad de Fortaleza, en un hospital universitario, cuyo objetivo fue conocer cómo los pacientes interpretan los cuidados de enfermería. En una de las conclusiones resaltan la importancia que la enfermera piense y tenga presente, que los cuidados brindados deben estar mediados por la preocupación y el desvelo por el otro, considerando este momento de comunión que será entre aquel que humana y, científicamente, aprendió los medios de mirar a otro en sus necesidades de salud y enfermedad.

Garzón (1998) afirma que la humanización comprende también los aspectos culturales, históricos y espirituales de hombre, para lo cual los profesionales de la salud deben establecer relaciones terapéuticas adecuadas. El mismo autor asegura que los humanistas de todas las corrientes filosóficas proclaman la dignidad del hombre, su libertad y la igualdad de derechos; se hace énfasis en la necesidad del amor y la fraternidad para gozar el derecho al bienestar, la paz y el progreso; se solicita el respeto mutuo para la convivencia, se invita al diálogo $\mathrm{y}$ al respeto del otro como interlocutor.

ACOFAEN (1998) afirma que el profesional de enfermería desarrolla su trabajo dentro de una relación privilegiada Enfermera - Paciente (familia, grupo), ofrece continuidad, brinda confianza e intimidad y conoce las necesidades de la persona, tanto aquellas derivadas del estado de salud o enfermedad como de otras situaciones de angustia y de preocupación que alteran su bienestar.

Según la ley 911, artículo 3 (2004), Tribunal Nacional Ético de Enfermería, el acto del cuidado de enfermería se da a partir de la comunicación y de la relación interpersonal humanizada entre el profesional de enfermería y el ser humano, sujeto de cuidado, su familia o grupo social en las distintas etapas de la vida, su situación de salud y del entorno, lo que implica un juicio de valor y un proceso dinámico y participativo para identificar y dar prioridad a las necesidades y decidir el plan de cuidados de enfermería.

De acuerdo a lo anterior, el cuidado de enfermería que hace énfasis en la humanización va más allá de realizar un simple procedimiento, implica estar atentos a las necesidades individuales y dirigirse a las madres como seres humanos, quienes poseen una serie de experiencias acumuladas durante toda su vida y que fueron aprendidas directamente o trasmitidas de generación en generación. Estas personas están inmersas en una sociedad y en un medio ambiente que, probablemente, condiciona su comportamiento y su respuesta durante el trabajo de parto y parto.

La humanización del cuidado en enfermería permite reflexionar sobre las necesidades de las mujeres que se encuentran en trabajo de parto y parto, las cuales deben ser atendidas con especial consideración por su estado de vulnerabilidad. Según la Declaración de Ceará (2002), en Fortaleza Brasil, se considera que el parto y el nacimiento son el principio y punto de partida de la vida y son, por ello, procesos que afectan al resto de la existencia humana. Según Vanegas (1995), para que los resultados del parto se lleven a cabo en forma exitosa, el personal de salud que atiende a la madre y a su hijo debe asumir una alta responsabilidad que implica sus acciones, sus omisiones y su actitud humanizada en este proceso. La madre y su hijo son seres holísticos, con percepciones y sensaciones estrictamente individuales.

La Red Latinoamericana y del Caribe para la humanización del parto y nacimiento RELACAHUPAN (2003) elaboró unos pasos o etapas que deberán seguirse en todos los países de la región, los cuales contemplan: el respeto a los derechos de la mujer y a su rol protagónico, erradicación de todas las formas de violencia en la atención, fomento y creación de mecanismos de denuncia, ofrecer espacios para la participación activa de la mujer en la atención materno-perinatal, revisión de tecnologías utilizadas y evaluación de las nuevas antes de su incorporación, capacitación permanente a las personas encargadas de los servicios de salud y atención con calidad sin 
discriminaciones, el compromiso y la dedicación serán aspectos fundamentales, sin dejar de lado el trabajo interdisciplinario que, según Andreoni et al. (2004), el profesional deberá estar abierto al diálogo, a los cambios, a compartir e incorporar nuevos conocimientos oriundos de otras disciplinas, provenientes de otros profesionales que hablan el mismo lenguaje.

Cuando se habla de humanización, no se pueden dejar de lado la aplicación de los aspectos éticos que deben estar presentes en los Profesionales de Enfermería que, teniendo en cuenta lo expuesto por Garzón (2005), a la persona se aprecia en el valor de sí mismo y en la ética de sus comportamientos e interrelaciones con los otros, con la familia, con la sociedad y con la comunidad.

\section{MATERIALES Y MÉTODOS}

Estudio de tipo prospectivo-descriptivo, se estudiaron a mujeres que concurrieron a una empresa Social del estado de II nivel de atención, en el Departamento de Cundinamarca, para la atención de parto. La muestra fue seleccionada por conveniencia, correspondiente a 80 mujeres que cumplieron con los siguientes criterios de inclusión: hospitalizadas en la sala de puerperio durante los meses de marzo a junio del 2005 y sin complicaciones durante el parto.

El periodo del estudio comprendió los años 2005 y 2006. Para la recolección de la información, se utilizó una encuesta semiestructurada, aplicada al grupo de mujeres en puerperio, previo consentimiento informado, en las que se incluyeron preguntas sobre la necesidad de acompañamiento de un familiar de apoyo durante el parto, identificación de la enfermera por parte de la gestante, forma de dirigirse, cuidados, actitud y comunicación de la enfermera con la familia de la gestante y privacidad. El instrumento que se aplicó fue previamente validado por cinco expertos, quienes recomendaron corregir preguntas que sugerían respuesta y organizar las preguntas por bloques temáticos. Posteriormente, se aplicó una prueba piloto a diez mujeres durante el trabajo de parto y parto, lo cual permitió aclarar algunas preguntas que eran incomprensibles.

Los resultados fueron analizados en porcentajes y se presentaron en gráficos de barras y diagramas de pastel.

\section{RESULTADOS Y DISCUSIÓN}

$\mathrm{Al}$ encuestar a las mujeres que se encontraban en puerperio en la institución donde se realizó el estudio y que habían tenido la experiencia del trabajo de parto y parto, específicamente en la variable relacionada con el deseo de las mujeres de ser acompañadas durante el parto, el dato más relevante, $51 \%$, hicieron referencia al deseo de haber sido acompañadas de su esposo o compañero, pero esta posibilidad no fue dada, ya que la institución hospitalaria no es establecido, como norma, el ingreso a la sala de partos a un familiar de apoyo. Al respecto, Monterrosa et al. (2006) citan a Hodnett, quien sugiere que el apoyo continuo durante el trabajo de parto debería ser la regla y no la excepción; según un estudio realizado por Arango et al. (2005), en las que se incluyeron a 1775 nacimientos ocurridos entre el primero de julio de 2004 y el 31 de marzo de 2005, el acompañamiento materno por parte de la pareja, un familiar u otra persona que no haga parte del equipo de salud es una práctica benéfica que debería ser rutinaria. Se demostró que las madres con apoyo continuo durante el trabajo de parto y parto requieren menos medicamentos para el alivio del dolor y sus recién nacidos requirieron menos maniobras de reanimación, cuando se comparan con mujeres atendidas con el cuidado usual. Además, es menos probable que se les realice episiotomía y perciben la maternidad como una experiencia más positiva, pero las políticas institucionales de los centros de atención de mayor nivel de complejidad no permiten la presencia de familiares en las salas de trabajo de parto.

En relación a la persona que debe acompañar en el parto, Dickason et al. (1999) afirman que se ha defendido intensamente la participación activa del padre, como entrenador en la pareja y, en las situaciones en que no está disponible, se busca un sustituto. Al respecto, Monterrosa et al. (2006) afirman la importancia de aumentar el acceso al apoyo personalizado y continuo durante el trabajo de parto, alentando a las mujeres a que soliciten a un familiar o amigo que se comprometa a estar presente en el momento del parto y a los proveedores de salud a permitir esta práctica. Según Luque $\mathcal{E}$ Oliver (2005) refieren que sería preciso que los profesionales sanitarios cuestionaran la creencia de que el acompañante de la mujer durante el proceso debería ser siempre el marido y discutir abiertamente, optando por su presencia cuando la mujer lo perciba como una 
ayuda y deseo real del futuro padre y no de una moda o tendencia cultural del momento.

Sin embargo y adicional a lo que afirman los anteriores autores, los resultados del presente estudio muestran que el $24 \%$ les pareció mejor estar solas, inclusive afirmaron "que lo del parto es asunto de mujeres o en otras circunstancias les hubiera dado vergüenza que el esposo o compañero las observara en esta situaciones".

Respecto a la identificación de la enfermera por parte de las gestantes que ingresaron a la sala de partos, el $48 \%$ no la identificaron, hecho que no dio la posibilidad de conocer si recibieron o no cuidados por parte de ella, pero hay que tener en cuenta que aspectos como la ropa que se usa en la sala de partos, tapabocas, guantes y gorro, de alguna forma interfieren en la identificación de la persona que está en contacto con la gestante; posiblemente la enfermera estuvo presente y brindó cuidados pero no fue posible conocer los cuidados dados por ellas. En un estudio realizado por Heluy et al. (2004) encontraron que los pacientes que asistieron a la sala de quirófano desconocían el nombre del profesional de enfermería que les atendía; todos los entrevistados reconocían que el personal que les prestaba asistencia era muy acogedor pero no conocían sus nombres, por lo tanto, la primera medida al entrar en contacto con las personas es identificarse con el nombre. Al respecto, Castañeda (1998) afirma que saludar y presentarse a la persona que ingresa al centro hospitalario permite establecer una buena relación de ayuda; la cortesía sincera y la sonrisa ofrecen acercamiento y seguridad para alguien que se enfrenta a una situación nueva, llena de incertidumbre, estrés y expectativa.

Así, se puede hablar que la enfermera trata a los sujetos de cuidado como personas; sin embargo, en la práctica diaria, Prieto (2000) menciona que en muchas situaciones el ser humano que requiere de nuestro cuidado pasa a ser "la historia 475", "el número 503", "la cama 8", "la toxémica". Al nominar a las personas por números o por otros denominativos, se despersonaliza y al despersonalizar, se deshumaniza. Los resultados del presente estudio mostraron que la enfermera se dirigió utilizando otros términos, el 58\% la llamaron "mamita"; al respecto, Castañeda (1998) señala la importancia de llamar a las personas por el nombre y no con calificativos estereotipados de presunta ternura, como madrecita, gordita, mi amor, entre otros.
En relación a la variable que hace referencia a los cuidados que recibieron las mujeres por parte de la enfermera, los resultados de la investigación muestran que el $73 \%$ percibieron algún tipo de cuidados directos, como son, administración de medicamentos, explicación de la forma de pujar y de respirar, brindar apoyo y explicación de procedimientos.

Si bien es cierto en la sala de partos la enfermera está pendiente de la madre respecto a múltiples necesidades, lo importante, según Reyes (2003) es el compromiso ante el que da y recibe el cuidado, el cual se percibe a través de fenómenos relacionados con la asistencia, técnicas adecuadas, manejo de tecnologías, capacidad para prevenir complicaciones, conductas de apoyo y actitudes cuidativas dirigidas a dignificar a otros en su condición humana para lograr un crecimiento integral. El estudio realizado por Daza $\varepsilon$ Medina (2006) muestran la trascendencia que debe tener la enfermera para valorar las necesidades básicas de las personas, utilizar el proceso de enfermería, como herramienta fundamental lo que le permitirá planear, organizar, ejecutar y evaluar el cuidado brindado a los pacientes y resaltar la importancia de brindar un cuidado personalizado. ACOFAEN (2000) afirma que la enfermera debe poner en práctica e introyectar los principios específicos que orientan la práctica de enfermería hacia la humanización en el cuidado de enfermería que son: la promoción de la calidad de vida, mayor interés por los derechos del paciente sin categorizarlo, incluir en los protocolos de cuidado de enfermería el componente humanístico, revisar las normas administrativas, tomar decisiones éticas, fortalecer el trabajo interdisciplinario hacia la humanización y profundizar en la dimensión humana del cuidado de enfermería, de tal manera que no solo se cumplan con una serie de procedimientos o actividades.

En un estudio fenomenológico realizado por Yajaira et al. (2002), se extractaron los enunciados de pacientes relacionados con la experiencia de cuidado humanizado y se destacan: a) los sentimientos del paciente, es decir, sentirse preciado, sentir compañía, sentirse informado y apoyado, de acuerdo a las características de la enfermera; b) ser cálida, cordial, oportuna y eficaz; c) brindar apoyo emocional, como acompañamiento en procedimientos y atención óptima. d) apoyo físico: tomar la mano y mirar al paciente; e) cualidades de la enfermera, expresadas en propiciar el diálogo y dar explicaciones con amabilidad. 
f) proactividad, estar dispuesta a dar una explicación anticipada y abogar por el paciente; g) empatía, como acercarse, brindar una relación cálida y ponerse en el lugar del otro; h) priorizar al ser, es decir, preocuparse por la persona y llamarla por el nombre, e i) disponibilidad de la atención: preguntar cómo está, cómo se siente, estar pendiente de las necesidades y estar pendiente del dolor. Otro estudio realizado por Balanza, citado por Beltrán (2006), en el que se buscaba comprender el significado del cuidado de enfermería para los pacientes hospitalizados en un servicio médico, encontró que para ellos, cuidado de Enfermería significaba ánimo, buen trato, amor y atención, que agradaba cuando se acompañaba de muestras de cariño.

Respecto a la actitud de la enfermera, en el presente estudio, se destaca que el 55\% del grupo de mujeres percibieron a la enfermera con una actitud comprensiva, seguida del 33\%, cariñosa. Watson (1989) complementa lo anterior afirmando que "la gente necesita de una persona amorosa y de cuidado"; si la humanidad va a subsistir, "se necesita volverse amorosos morales y cuidadores, se necesita amor, respeto, cuidar de sí mismos y tratarse con dignidad antes de respetar amar y cuidar a otros y tratarlos con dignidad", y si de leyes se trata, según el Tribunal Nacional de Ética en Enfermería, ley 911, artículo 14, se describe que la actitud de la enfermera será de apoyo, prudencia y adecuada comunicación e información, adoptará una conducta respetuosa y tolerante frente a las creencias, valores culturales y convicciones religiosas de los sujetos de cuidado.

Otro aspecto a tener en cuenta, según Rojas \& Navarro (2006), es la angustia, el dolor, la ansiedad, la debilidad y el desespero, cuando un individuo se encuentra en un centro asistencial, ya que se crea una carga de estrés difícil de sobrellevar, dando como resultado la concepción de una idea macabra, abominable e injusta de lo que ya se está viviendo, entonces, el campo de acción de la profesión de enfermería, no se limita a la aplicación de tratamientos rutinarios o cumplimiento de ordenes clínicas, sino, por el contrario la labor comienza desde el mismo instante en que el paciente busca ayuda, porque es la forma de hablar, la manera de mirar, la postura y la disposición para escuchar, las que hacen posible que esa sensación de miedo se haga más leve.

Respecto a la comunicación de la enfermera con la familia de la gestante, el mayor porcentaje de mujeres, $63 \%$, manifiestaron que la enfermera no estuvo con su familia. Estos resultados permiten concluir que las enfermeras no destinaron tiempo para esta actividad y es preciso recordar que desde el momento que la mujer ingresa a la sala de trabajo de parto, pierde el contacto directo con sus familiares; la intervención de la enfermera, en este momento, juega un papel importante para identificar las necesidades de la familia, realizar las respectivas intervenciones y reconocer que es un derecho que tienen los familiares de ser informados sobre la situación de la mujer gestante. Cabe también recordar que el tiempo de duración en un trabajo de parto es relativo y depende de las condiciones específicas de la madre o de complicaciones que se pueden presentar, entonces, son los familiares quienes con ansiedad esperan apoyo e información oportuna.

En cuanto a las preguntas relacionadas con el mantenimiento de la privacidad, tono de voz y comentarios inapropiados y exposición de su cuerpo de manera innecesaria durante el trabajo de parto y parto, el $100 \%$ de las mujeres consideraron que no hubo problemas al respecto, por el contrario generalizaron haberse sentido bien con todo el personal que estuvo presente; ninguna de las mujeres encuestadas hizo especificaciones sobre la enfermera, inclusive algunos comentarios de ellas fueron los siguientes: "me realizaron tactos ante varias personas pero con los dolores no me sentí incómoda" y otra afirmó que estuvo expuesto su cuerpo ante varias personas pero no se sintió molesta. Sin embargo, contrario a lo que se encontró en la presente investigación, Pupulim E Sawada (2002) afirman que los enfermeros constantemente invaden la intimidad y la privacidad del paciente al realizar los cuidados de enfermería y raramente discuten los aspectos que involucran este problema. El estudio también tuvo como objetivo sensibilizar a los profesionales en enfermería al respecto de la relevancia y la necesidad de reflexión sobre este asunto; se resaltaron los aspectos ético-legales y morales involucrados en la invasión de la privacidad, apuntando las responsabilidades de estos profesionales. Se discute la actuación de los Comités de Ética en Investigación y de las Comisiones de Ética de Enfermería de las entidades, como mecanismos de control y protección de los individuos, ya que la protección de la privacidad de enfermos todavía es deficiente.

De acuerdo a lo anterior cabe destacar que el tema de la privacidad, derechos de los pacientes y aspectos ético 
legales en el cuidado de enfermería, siempre deben estar presentes. La enfermería tiene un rol especial y es el de estar atentos a la supervisión permanente de estos aspectos, ya que podrían existir situaciones en las que las mujeres, por el hecho de estar con dolor y con la expectativa de que todo el proceso del parto salga bien, no puedan realmente percibir si se da o no cumplimiento al mantenimiento de la privacidad y respeto por ellas.

Con base en los resultados, se elaboró un modelo de cuidado humanizado, en el que se describe inicialmente la importancia que el profesional de enfermería conozca las condiciones socio-demográficas y situaciones que vivencia una mujer en el momento de su ingreso a la sala de partos (figuras 1 y 2); dichas condiciones y situaciones pueden hacer que las mujeres actúen de manera distinta e influir en los resultados del trabajo de parto. De igual manera, cada una de las mujeres tienen necesidades diferentes (figura 3), para lo cual, se requiere que el profesional de enfermería asegure su atención, teniendo en cuenta sus individualidades y, además, posea unas actitudes (figura 4) que le permitan brindarl seguridad, tener una experiencia agradable para sus vidas y que cuenten con el apoyo incondicional, para que este proceso se pueda dar lo mejor posible en medio de las posibles dificultades o situaciones de estrés que se puedan generar durante el parto. Si la enfermera pone en práctica dichas actitudes, se facilita la interacción, la comunicación, la expresión de necesidades e inquietudes de las personas y, a la vez tener la oportunidad de vivenciar algo muy grande en el cuidado de enfermería que es presencia auténtica, dejando a un lado la rutina y pasar a un nivel superior de interacción con las personas a su cuidado.

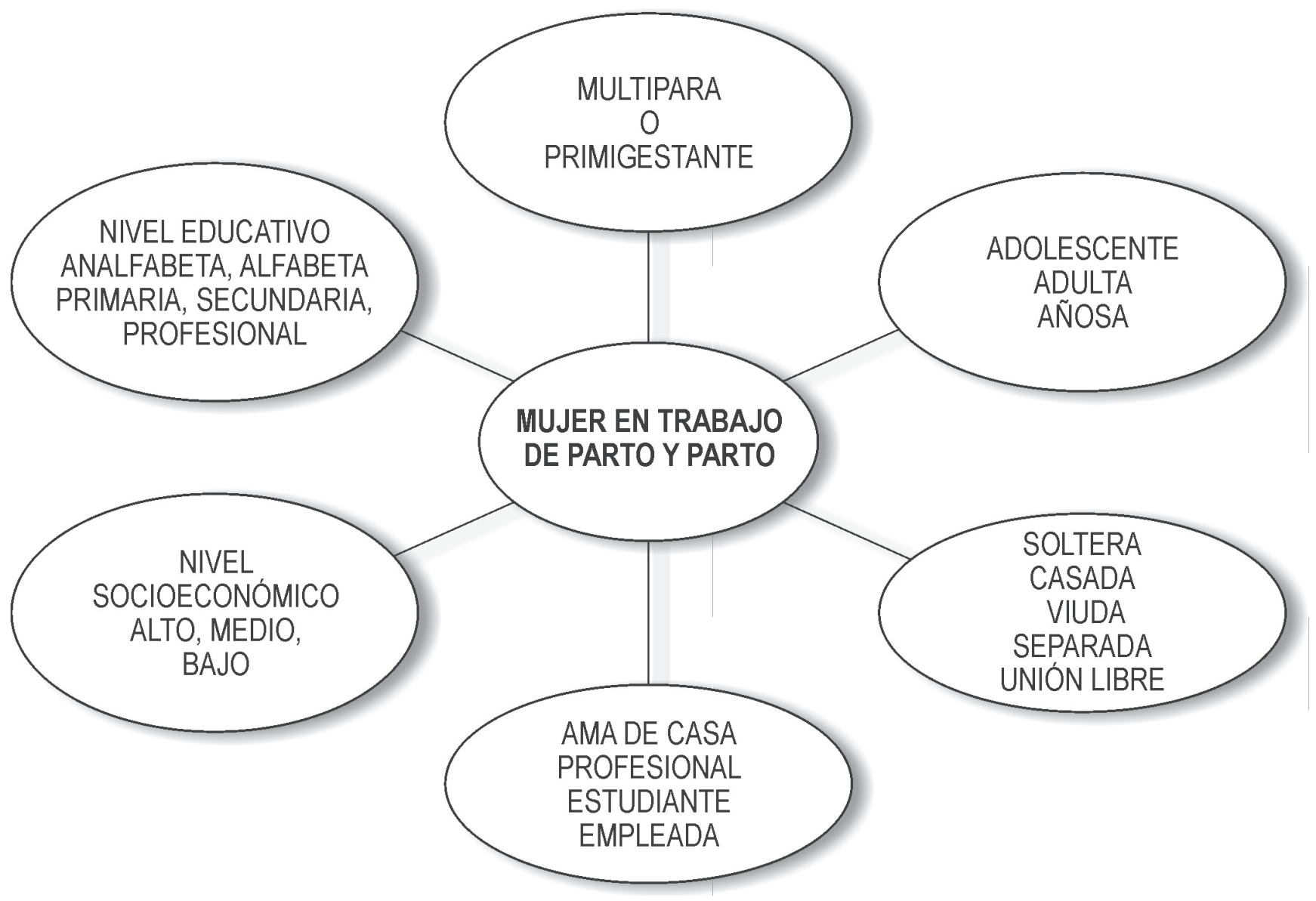

Figura 1. Condiciones socio-demográficas de las mujeres que ingresan a una sala de partos. 


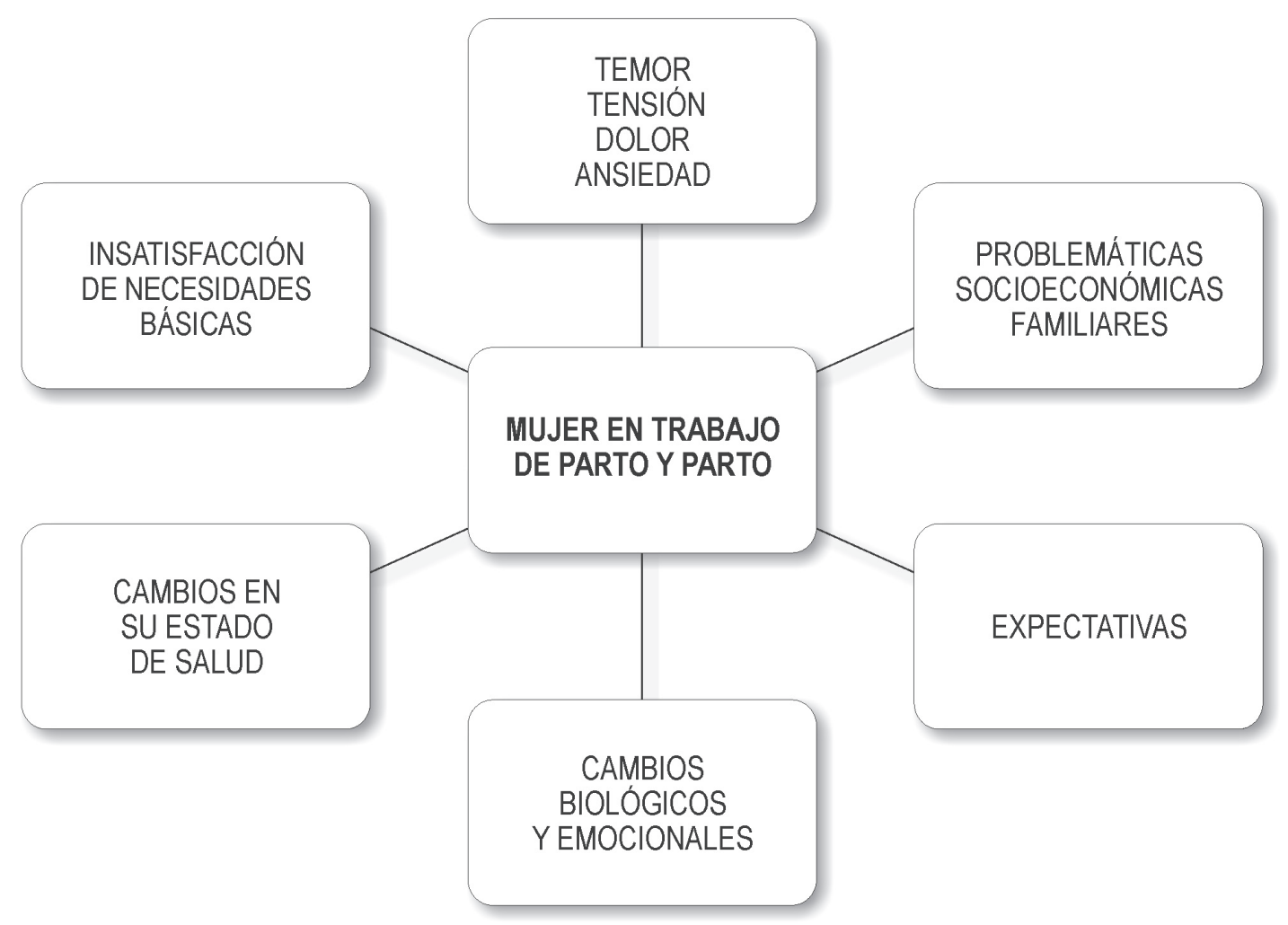

Figura 2. Situaciones que vivencia una mujer cuando ingresa a una sala de partos.

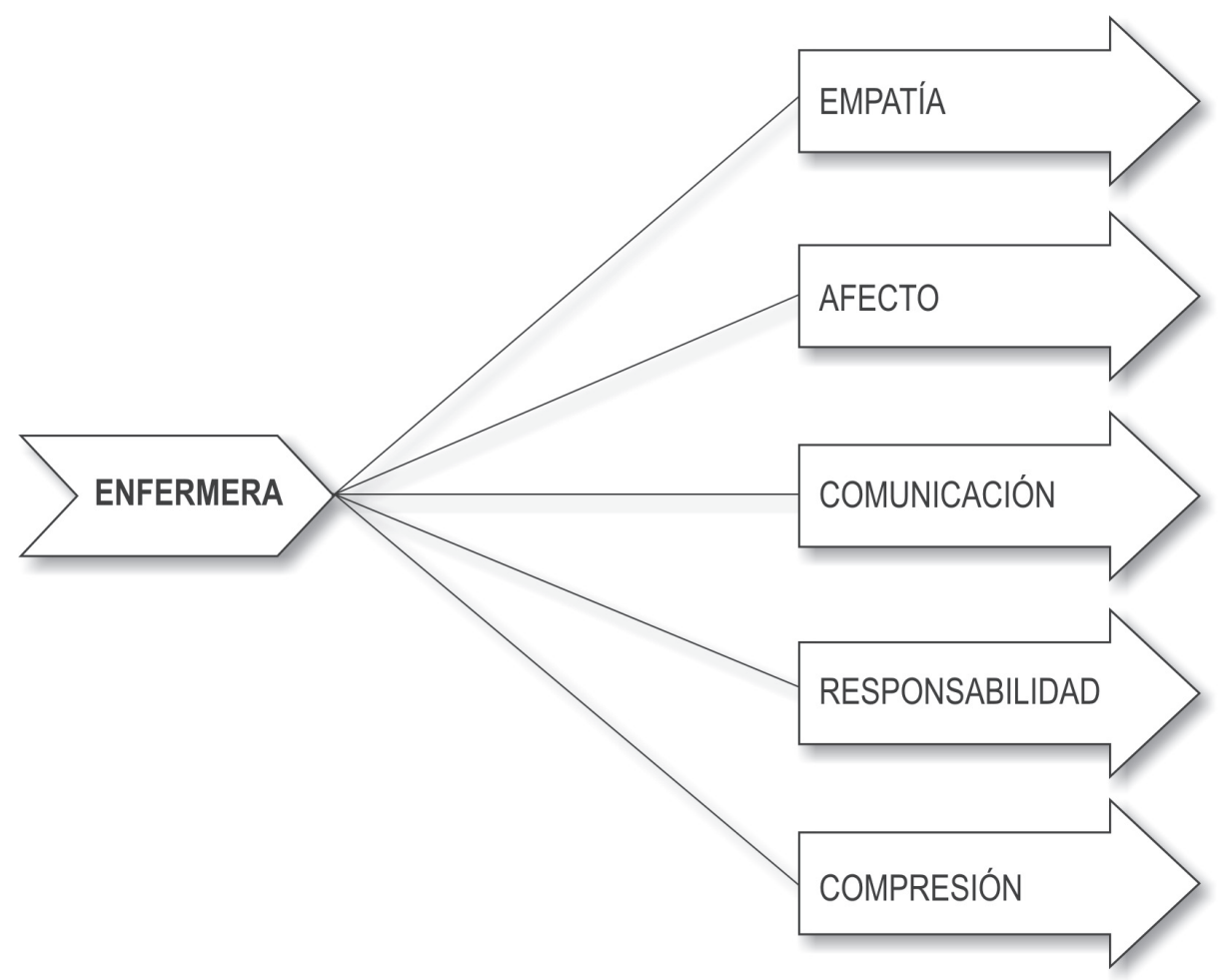

Figura 3. Actitudes de la enfermera y la humanización del cuidado a la mujer durante el parto. 


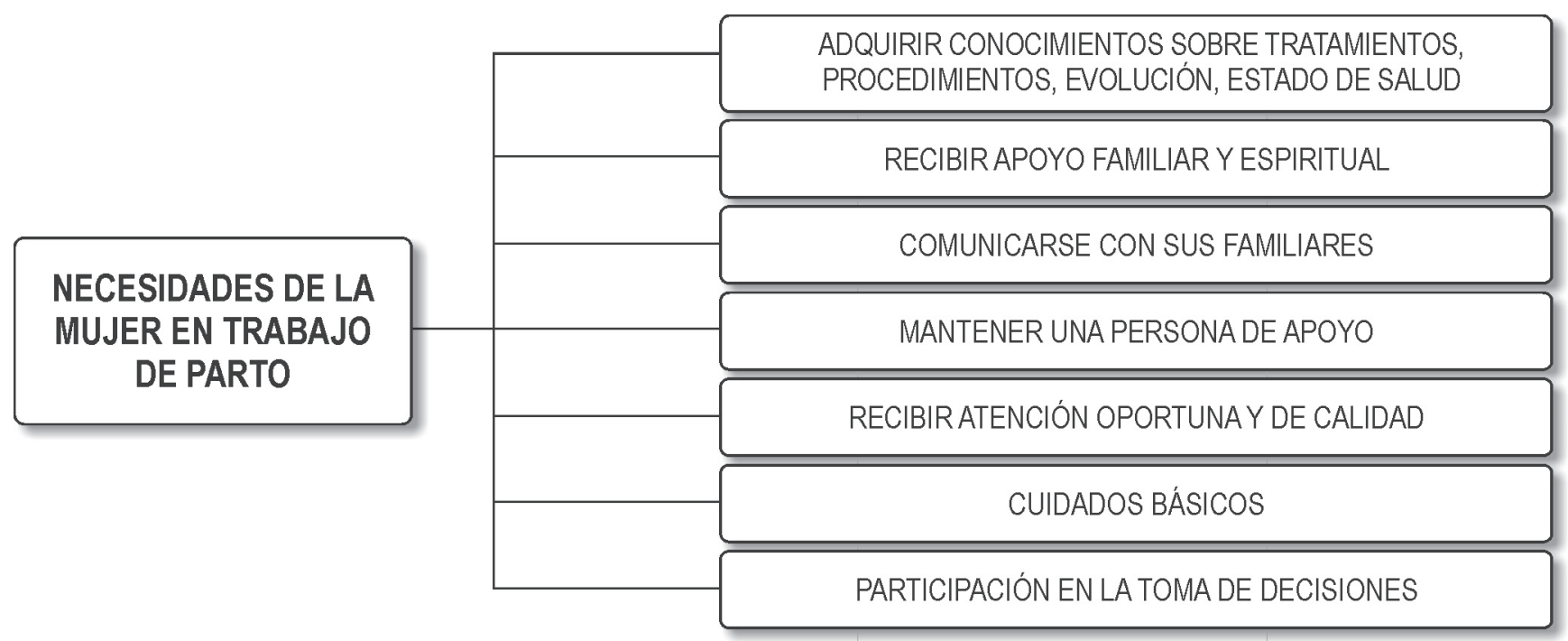

Figura 4. Necesidades de la mujer en trabajo de parto y parto.

Si bien es cierto que la enfermera además de poner en práctica las actitudes de cuidado, debe cumplir con otras funciones especificas, lo cual no es posible lograrlo si no se cuenta con una adecuada gestión administrativa y de recursos (figura 5), para el logro de los objetivo que se pretende, como es el de dimensionar el cuidado para fortalecer y dignificar cada día más la misión del profesional de enfermería en esta área.
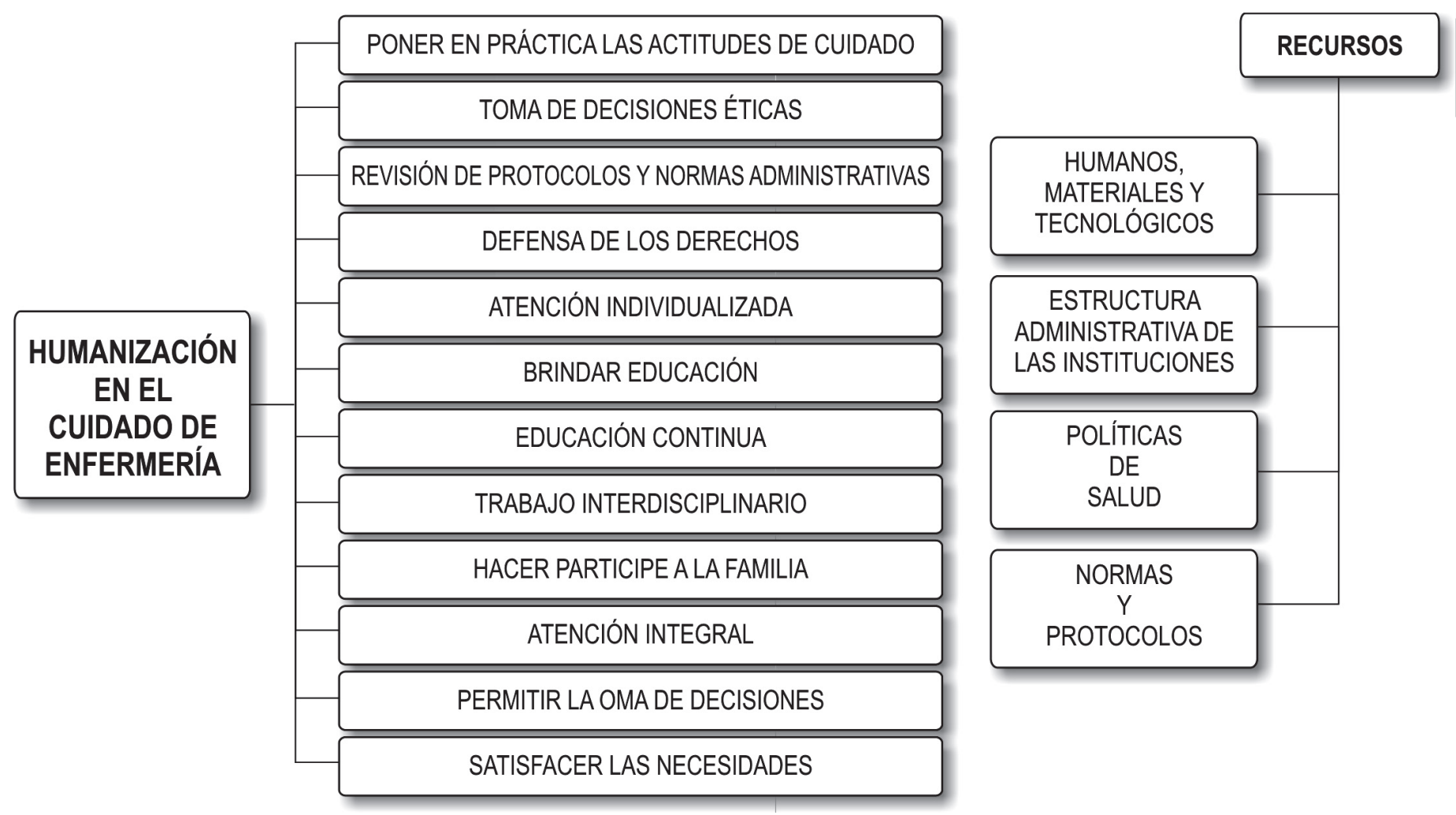

Figura 5. Implicaciones de enfermería en la humanización del cuidado. 


\section{CONCLUSIONES}

La planeación del cuidado de enfermería a las mujeres durante el parto debe ser individualizado, lo cual permite asegurar la satisfacción de sus necesidades.

Las mujeres que ingresan a una sala de partos de la institución donde se realizó el estudio, manifestaron requerir acompañamiento durante el parto; sin embargo, existen diferencias dependiendo de su propia percepción acerca del parto.

La humanización en el cuidado de enfermería implica, para el profesional, la aplicación de conocimientos científicotécnicos, un trato amable y la aplicación de actitudes cuidativas que permitan el crecimiento en el cuidado, a través de la interacción y la comunicación con los otros.

La familia de la mujer que se encuentra en trabajo de parto también necesita de apoyo, de educación y de comprensión, enfermería debe participar en la intervención de estos cuidados.

La enfermera debe estar siempre atenta al mantenimiento del respeto y la privacidad de las personas a quien dirige el cuidado, no solo por parte de ella, sino de todo el personal que entra en contacto con una mujer durante el trabajo de parto y parto.

\section{BIBLIOGRAFÍA}

ACOFAEN. 1.998. Código de Ética de Enfermería. (Bogotá Colombia). 7p.

ACOFAEN. 2000. Ética y enfermería. (Bogotá. Colombia).7p.

ANDREONI, S.; BRUGGEMANN, O.M.; CAMACHO, A.V.; CIANCIARULLO, T.; LAND, S.; TSUNECHIRO, M.A.; GÓMEZ, E. 2004. Lineamientos y Directrices de Enfermería Para la Mejoría de la Calidad de la Atención Prenatal en Embarazos de Bajo Riesgo en América Latina y el Caribe, OPS. (Washington, D.C) p.7-42. Disponible desde Internet en: http:// www.paho.org/Spanish/AD/FCH/WM/PrenatalcareManualesp.pdf (con acceso 20/10/07).

ARANGO, F.; GÓMEZ, J.C.; ZULETA, J.J. 2005. Uso de prácticas clínicas durante el embarazo, parto, puerperio y recién nacido, en hospitales públicos de Manizales. Rev. Col. Obst. Ginecol. 56(4):271280.

BELTRÁN, O.A. 2006. Factores que Influyen en la interacción humana del cuidado de enfermería. Investigación y Educación en Enfermería. U. Antioquia. (Colombia). 24(2):144-150.

CASTAÑEDA, E. 1998. La enfermera y la humanización de la atención a la salud. Hacia una medicina más humana. Ed. Médica Panamericana. (Bogotá). 57-59.

DAZA, R.; MEDINA, L.S. 2006. Significado del cuidado de enfermería desde la perspectiva de los profesionales de una institución hospitalaria de tercer nivel en Santafé de Bogotá, Colombia. Cultura de los cuidados. Rev. Enfermería y Humanidades. U. Alicante (España). 10(19):55-62.

DA SILVA, L.; COELHO M.; DE LIMA C.; SILVA,PAULA. 2002 Cuidados De Enfermería su Sentido para Enfermera y Pacientes. Rev. Enfermería Chile. 120(2):22-28.

DECLARACIÓN DE CEARÁ. 2002. Sobre la humanización del parto, (Fortaleza. Brasil). Disponible desde Internet en:

http://www.relacahupan.org/caminos01.html (con acceso el 04/15/07).

DICKASON, E.; LANG, B.; KAPLAN, J. 1999. Enfermería materno infantil. Serie Mosby de Enfermería Clínica. Ed: Harcourt (Madrid). p.3.

GARZÓN, N. 1998. Ciencia y humanización en enfermería. En: Quevedo, F.; Plata, E.; Mendoza, J. Hacia una medicina más humana. Ed Médica Panamericana. (Bogota, Colombia). p.248-250.

GARZÓN, N. 2005. Ética profesional y teorías de enfermería. Aquichan. (Bogotá, Colombia). 5(1):69-71.

HELUY, C.; DE FARIA, T.E, CABAÑERO, R.F., CABO, M.C. 2004 Humanización de la atención de enfermería en el quirófano. Index Enferm. (España) 3 (44-45):18-20. Disponible desde Internet en: 
http://scielo.isciii.es/scielo.php?script $=$ sci arttextEpid=S1132-12962004000100004Elng $=$ esEnrm $=$ iso (con acceso el 03/12/07).

LUQUE, M.; OLIVER, M. 2005. Diferencias culturales en la percepción y vivencia del parto en el caso de las mujeres inmigradas. Index Enferm. (España) 14:48-49. Disponible desde Internet en: http://wwwscielo.isciii.es/scielo. php?script $=$ sci_arttextEpid $=$ S1132-1296200 $5000100002 \varepsilon \operatorname{lng}=\mathrm{es} E \mathrm{nrm}=\mathrm{iso}($ con acceso 21/10/07).

MONTERROSA, E.; ZULETA, J.; ROJAS, R. 2006. Prácticas basadas en la evidencia en el cuidado de la gestación trabajo de parto, puerperio y recién nacido. Avances en Enfermería (Colombia). 24(1):43-52.

PRIETO, G.; 2000. Humanización del cuidado de enfermería en ética y enfermería. Boletín Latinoamericano 4. Comité de Ética. ACOFAEN. Bogotá. p.7

PUPULIM, J.; SAWADA, N. 2002. Nursing care and the invasion of patients' privacy: an ethical and moral issue. Rev. Latino-Am. Enfermagem 10(3):433-438. disponible desde Internet en : $<$ http://www.scielo.br/scielo.php?script $=$ sci arttextEpid=S0104-11692002000300018Elng = enEnrm=iso >. ISSN 0104-1169. doi: 10.1590/ S0104-11692002000300018 ( con acceso 24/03/08).

RELACAHUPAN. 2003. Caminos para la humanización del parto y el nacimiento. Disponible desde Internet en: http://www.relacahupan.org/caminos01. html (con acceso 29/11/07).
REYES, I. 2003. Relación filosófica de las teorías de enfermería (Venezuela). Disponible desde Internet: en: http://es.geocities.com/mi_portal_de_en-

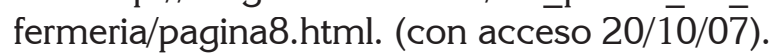

ROJAS, E.; NAVARRO, D. 2006. El sentido humano entre enfermera y paciente. Rev. Ciencia y Cuidado (Colombia). 3(3):37-42.

TREVIZAN, M.A; MENDES, I.A; MELO, M.R. 2003. Al encuentro de la competencia del cuidado según Boff, una nueva perspectiva de conducta ética de la enfermera gerente. Rev. Latino-Americana de Enfermería. 11(5):652-657. Disponible desde Internet en: <http://www.scielo.br/scielo.php?script = sci arttextEpid=S0104-11692003000500013Elng = esEnrm=iso (con acceso 20/10/07).

TRIBUNAL NACIONAL ÉTICO DE ENFERMERÍA. Ley 911. 2004. (Bogotá). p.5-20.

VANEGAS, B.C. 1995. Fisiología del parto. Unidad de autoaprendizaje Ed. Unibiblos. (Bogotá). p.77-83.

WATSON, J. 1989. Nursing: human science and human care. a theory of nursing. Norwalk, CT: Appleton - Century - Crofts. (New York). 20p.

YAJAIRA, M.; MUÑOZ, S.; ALVIS, T. 2002. Experiencia de recibir un cuidado de enfermería humanizado en un servicio de hospitalización. En: El arte y la ciencia del cuidado. U. N. Colombia, Facultad de Enfermería. Ed. Unibiblos (Bogotá-Colombia). p.214-215.

Recibido: Junio 6 de 2007

Aceptado: Abril 9 de 2008 